of Medicine, Beni-Suef University, Beni-Suef, ${ }^{7}$ Internal Medicine, Rheumatology Unit, Egyptian Atomic Energy Authority (EAEA), Cairo, ${ }^{8}$ Rheumatology, Faculty of Medicine, Fayoum University, Fayoum, ${ }^{9}$ Rheumatology, Faculty of Medicine, Minia University, Minia, ${ }^{10}$ Rheumatology, Faculty of Medicine, Beni-Suef University, Beni-Suef, ${ }^{11}$ Internal Medicine, Rheumatology Unit, Faculty of Medicine, Cairo University, Cairo, ${ }^{12}$ Rheumatology, Faculty of Medicine, Suez-Canal University, Ismailia, ${ }^{13}$ Rheumatology, Faculty of Medicine, Ain-Shams University, Cairo, ${ }^{14}$ Ophthalmology, Faculty of Medicine, Beni-Suef University, Beni-Suef, Egypt

Background: Behçet's disease is a chronic, relapsing multisystemic vasculitis that is common in the Mediterranean basin region with a diverse spectrum of clinical manifestations. A previous single-centre study from Alexandria conducted 20 years ago reported a prevalence of 7.6/100.000 population and a M:F ratio of $5.4: 1$.

Objectives: The aim of this multicenter study was to present the prevalence and pattern of Behcet's disease (BD) in adult Egyptian patients.

Methods: This study included 1098 BD patients from 14 highly specialised centres over. Egypt during 2017 The demographic, clinical characteristics, laboratory findings, $B D$ current activity form (BDCAF) and medications received were reported.

Results: The mean age of the patients was $36.3 \pm 10.2$ years (16-73 years), disease duration was $6.7 \pm 5.2$ years and they were 776 males and 322 females (2.4:1) and was 1.9:1 from Alexandria. Their mean age at disease onset was 29.8 \pm 8.7 years. $24.9 \%$ were diabetic and $30.7 \%$ hypertensive. The frequency of presenting cases was highest from Alexandria (41.3\%) followed by Cairo (35.3\%), Beni-Suef (6.4\%), Assuit (6.1\%), Sharkia (3.9\%), Fayoum (3.2\%), Minia (2.7\%) and $1.1 \%$ from Ismailia. The total prevalence from those 8 major cities after adjusting for adults according to the 2017 population consensus was $4.35 / 100.000$ inhabitants; 13.76 for Alexandria, 6.3 for Cairo and the highest prevalence in upper Egypt from Beni-Suef was 3.48 and the least from Minia (0.85). The prevalence from Sharkia was 0.93, Fayoum 1.51 and was 1.41 from Ismailia. All patients had oral ulcers while genital ulcers were present in $80 \%$. The eyes were involved in $78.9 \%$, cutaneous manifestations were present in $59.8 \%$, arthritis in $34.7 \%$, neuropsychiatric manifestations in $13.9 \%$, gastrointestinal tract involvement in $11.5 \%$ and deep venous thrombosis in $23.1 \%$. Their mean BDCAF was 4.6 \pm 4 .6. The mean erythrocyte sedimentation rate was $29.1 \pm 19.9 \mathrm{~mm} / 1 \mathrm{sthr}, \mathrm{C}$ reactive protein $11.3 \pm 15.2 \mathrm{mg} / \mathrm{dl}$, haemoglobin content $13.3 \pm 6 \mathrm{~g} / \mathrm{dl}$, total leucocytic count $8.6 \pm 14.5 \times 10^{3} / \mathrm{mm}^{3}$, platelets $262.6 \pm 76.9 \times 10^{3} / \mathrm{mm}^{3}$ and serum uric acid $4.8 \pm 1.6 \mathrm{mg} / \mathrm{dl}$. $90.7 \%$ were receiving steroids, $81.1 \%$ were receiving colchicine, while only $8.1 \%$ were receiving biologic therapy.

Conclusions: The prevalence of BD in Egypt is high and higher in Alexandria than previously reported. The male-to-female ratio is lower than previously found being more in harmony with the globally reported ratios. The pattern of clinical presentation is unique for this country yet comparable to the universally stated frequencies.

Disclosure of Interest: None declared

DOI: 10.1136/annrheumdis-2018-eular.4833

\section{AB1349 SAFETY AND EFFECTIVENESS OF COSMETIC MINIMALY INVASIVE PROCEDURES AMONG PATIENTS WITH SYSTEMIC AUTOIMMUNE DISEASE}

O. Reitblat, T.T. Lerman, T. Reitblat. Rheumatology, Barzilai MC, Ashkelon, Israel

Background: Noninvasive or minimally invasive cosmetic dermatologic procedures are considered safe with low parentage of reported adverse events. However, reliable prevalence data regarding adverse event of such cosmetic procedures among patients with systemic autoimmune diseases are insufficient. Objectives: To assess the occurrence of adverse events and disease exacerbation associated with noninvasive or minimally invasive cosmetic dermatologic procedures, including those involving hyaluronic acid fillers, botulinum toxins and laser application among patients with systemic autoimmune diseases.

Methods: Consecutive cases of patients suffering from autoimmune diseases and attending the rheumatology clinic for regular follow-ups, were asked about receiving cosmetic procedures during the last two years. Medical history, including clinical and laboratory signs of disease exacerbation after the date of the procedure, was retrospectively obtained from medical files of the patients included in the study. Patients were also asked about subjective disease exacerbation and local adverse events after the procedure.

Results: During the three months of study period, 148 patients were inquired. Nineteen patients (89\% females) underwent 23 cosmetic procedures in total. Thirty-nine percent had Rheumatoid arthritis (RA), 39\% had Ankylosing spondylitis (AS) and $22 \%$ had other systemic connective tissue disease. Sixty seven percent were treated by Disease-modifying antirheumatic drugs (DMARDs), $28 \%$ by Biologic treatment and $5 \%$ did not receive any specific treatment. All patients were in remission during the cosmetic procedures. Forty three and a half percent of patients underwent hyaluronic acid injection, $21.7 \%$ botulinum toxin injection, $21.7 \%$ laser application, $8.7 \%$ mesotherapy and $4.3 \%$ silicon injection. None of the patients suffered from subjective disease exacerbation after the procedure. No changes in antibody titer and level of acute phase reactants (C-reactive protein and erythrocyte sedimentation rate) were noticed. Two patients (10.5\%) experienced local oedema after filler injections. Both patients received Hydroxychloroquine treatment (one patient with RA and one with AS).

Conclusions: Our results suggest that noninvasive or minimally invasive cosmetic dermatologic procedures, including energy, neurotoxin, and filler procedures, may be safe among rheumatological patients, and do not cause autoimmune systemic disease exacerbation when performed in periods of remis sion. Hydroxychloroquine may predispose to a higher occurrence rate of local site injection adverse events. Further studied are desired to investigate this phenomena.

Disclosure of Interest: None declared

DOI: 10.1136/annrheumdis-2018-eular.1093

\section{AB1350 IS THE RADIOGRAPHIC DAMAGE A RISK FACTOR FOR} NEUROPATHIC PAIN IN RHEUMATOID ARTHRITIS?

T.M. Rocha ${ }^{1}$, S. Pimenta ${ }^{1}$, M. Bernardes ${ }^{1}$, A. Bernardo ${ }^{1}$, M. Barbosa ${ }^{2}$, R. Lucas ${ }^{3}$, L. Costa ${ }^{1} .{ }^{1}$ Rheumatology; ${ }^{2}$ Pain Unit, Centro Hospitalar de São João; ${ }^{3}$ Clinical Epidemiology, Faculty of Medicine University of Porto (FMUP), Porto, Portugal

Background: Features suggestive of neuropathic pain (NP) have been described in Rheumatoid Arthritis (RA) patients. The structural damage assessed on radiographs is a direct consequence and reflection of cumulative of disease activity and its association with NP has not been studied in RA.

Objectives: To determine whether existence and intensity of NP is associated with radiographic damage in $\mathrm{RA}$ patients.

Methods: Cross-sectional study was performed with RA patients followed at our Rheumatology department. Patients with diagnosed neuropathy or non-RA risk factors for NP were excluded. Selected patients were evaluated in a medical visit. Two questionnaires were applied to assess NP: the Leeds Assessment of Neuropathic Symptoms (LANSS) and the painDETECT (PDQ). Wrist, hands and feet radiographic studies from the previous 12 months before were classified according to the modified van der Heijde Sharp's method by one trained reader, blinded for patient clinical variables and treatment allocation. Correlation studies (spearman coefficient analysis) and univariate and multivariate logistic regression were performed. Significance level was set as $<0.05$.

Results: Ninety one RA patients were included. Seventy (77\%) were women with a mean age of $55.6 \pm 10.8$ years and median disease duration of 12 years; ${ }^{2-4}$ 84\% patients were seropositive for Rheumatoid Factor and/or ACPA; 85 (93\%) were treated with conventional synthetic Disease-Modifying Antirheumatic Drugs (DMARDs) and $41 \%$ with a biological DMARD (bDMARDs). The mean DAS28 4V $\mathrm{CRP}$ was $3.15 \pm 0.77$ and the mean $\mathrm{HAQ}$ score was $1.04 \pm 0.6$. The median joint erosion score (JE) was $28^{3-143}$ and the median joint space narrowing (JN) was 46. ${ }^{10-133}$ Forty-two (46\%) patients had LANSS NP $(\geq 12), 29 \%$ had a possible/ likely NP in the PDQ (>12), and 13\% had likely NP in the PDQ $(>18)$. JN and global score (GS) had a negative weak correlation with LANSS ( $r=-0.21$ and $r=-0.25$, respectively, $p<0.05)$ and $J N$ correlated with $P D Q(r=-0.23, p=0.03)$. No significant correlations were observed with JE. Disease duration significantly correlated with all the radiographic scores ( $r=0.48$ for $G S, r=0.43$ for $J N, r=0.44$ for JE, $\mathrm{p}<0.05)$ and negatively correlated with LANSS $(r=-0.28, p=0.01)$. Lower median GS values were observed in LANSS positive group (62 vs 79, $p=0.01$ ). Patients under bDMARDs had significant higher median $G S$ ( 80 vs $61, p=0.03$ ) but also higher disease duration ( 14 vs $10, p=0.01$ ). No statistically significant differences were observed for other variables. Disease duration was a negative predictor of LANSS NP (OR: 0.98, $\mathrm{p}=0.03$ ). JN was inversely associated with LANSS NP (OR: 0.978, $p=0.02$ ) and remained significant after adjustments for bDMARDs treatment, but not for disease duration. JN was a also a negative predictor of PDQ likely NP and remained significant after adjustment for bDMARDs (OR: 0.979 $\mathrm{p}=0.03$ ), but not for disease duration.

Conclusions: In this cohort, JN score had a weak negative association with NP. Higher structural damage and disease duration do not seem to increase the risk of non-nociceptive RA pain. Further studies are needed to confirm these results.

\section{REFERENCES :}

[1] Koop SM, et al. Arthritis Res Ther. 2015; 17:237

[2] van der Heijde D. J Rheumatol 1999;26:743-5.

Disclosure of Interest: T. Rocha Grant/research support from: Portuguese Society of Rheumatology/Alfa Wassermann on May 2015, S. Pimenta: None declared, M. Bernardes: None declared, A. Bernardo: None declared, M. Barbosa: None declared, R. Lucas: None declared, L. Costa: None declared DOI: 10.1136/annrheumdis-2018-eular.5653 